\title{
FRAGMENTS OF FIRST-ORDER LOGIC OVER INFINITE WORDS (EXTENDED ABSTRACT)
}

\author{
VOLKER DIEKERT $^{1}$ AND MANFRED KUFLEITNER ${ }^{1}$ \\ ${ }^{1}$ Universität Stuttgart, FMI, Universitätsstraße 38, D-70569 Stuttgart, Germany
}

\begin{abstract}
We give topological and algebraic characterizations as well as language theoretic descriptions of the following subclasses of first-order logic $\mathrm{FO}[<]$ for $\omega$-languages: $\Sigma_{2}, \Delta_{2}, \mathrm{FO}^{2} \cap \Sigma_{2}$ (and by duality $\mathrm{FO}^{2} \cap \Pi_{2}$ ), and $\mathrm{FO}^{2}$. These descriptions extend the respective results for finite words. In particular, we relate the above fragments to language classes of certain (unambiguous) polynomials. An immediate consequence is the decidability of the membership problem of these classes, but this was shown before by Wilke [20] and Bojańczyk [2] and is therefore not our main focus. The paper is about the interplay of algebraic, topological, and language theoretic properties.
\end{abstract}

\section{Introduction}

The algebraic approach for fragments of first-order logic over finite words has been very fruitful. For example, a result of Wilke and Thérien is that $\mathrm{FO}^{2}$ and $\Delta_{2}$ have the same expressive power [14], where the latter class by definition denotes $\Sigma_{2} \cap \Pi_{2}$. Further results are language theoretic and (very often decidable) algebraic characterizations of logical fragments, see e.g. [13] or [4] for surveys. Several results for finite words have been extended to other structures such as trees and other graphs, see [18] for a survey. For some characterizations over finite words, it has been shown that they cannot be generalized; e.g. over unranked trees, it turned out that $\mathrm{FO}^{2}$ and $\Delta_{2}$ are incomparable [1]. For infinite words, it is clear that the expressive power of $\mathrm{FO}^{2}$ is not equal to $\Delta_{2}$, since saying that letters $a$ and $b$ appear infinitely often, but $c$ only finitely many times is $\mathrm{FO}^{2}$-definable, but there is neither a $\Sigma_{2}$-formula nor a $\Pi_{2}$-formula specifying this language.

Our results deepen the understanding of first-order fragments over infinite words. A decidable characterization of the membership problem for $\mathrm{FO}^{2}$ over infinite words has been given in the habilitation thesis of Wilke [20]. Recently, decidability for $\Sigma_{2}$ has been shown independently by Bojańczyk [2]. Language theoretic and decidable algebraic characterizations of the fragment $\Sigma_{1}$ and of its Boolean closure can be found in $[8,9]$.

We introduce two generalizations of the usual Cantor topology for infinite words. One of our first results is a characterization of languages $L \subseteq \Gamma^{\infty}$ being $\Sigma_{2}$-definable in terms

Received by the editors December 16, 2008.

1998 ACM Subject Classification: F.4.1 Mathematical Logic, F.4.3 Formal Languages.

Key words and phrases: infinite words, regular languages, first-order logic, automata theory, semigroups, topology.

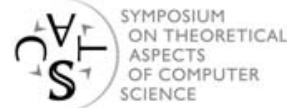


of a property of its syntactic monoid and by requiring that $L$ is open in some alphabetic topology. Both properties are decidable.

Our second result is that a language is $\mathrm{FO}^{2}$-definable if and only if its syntactic monoid is in the variety DA. (The result is surprising in the sense that it contradicts an explicit statement in [20]). Moreover, we show that $\mathrm{FO}^{2}$-definability can be characterized by being closed in some further refined alphabetic topology and in terms of weak recognition by some monoid in DA. In particular, weak recognition and strong recognition do not coincide for the variety DA. This seems to be a new result as well. We also contribute a language theoretic characterization of $\mathrm{FO}^{2}$ in terms of unambiguous polynomials with additional constraints on the letters which occur infinitely often.

Further main results of our paper are the characterization of $\mathrm{FO}^{2} \cap \Sigma_{2}$ as the class of unambiguous polynomials and of $\Delta_{2}$ in terms of unambiguous polynomials in some special form. In particular, it follows already from this description that $\Delta_{2}$ is a strict subset of $\mathrm{FO}^{2}$. Furthermore, we show that the equality of $\mathrm{FO}^{2}$ and $\Delta_{2}$ holds relativized to some fixed set of letters which occur infinitely often. If this set of letters is empty, we obtain the situation for finite words as a special case. Finally, we relate topological constructions such as interior and closure with membership in the fragments under consideration. Among other results, we are going to explain the following relations between the fragments $\mathrm{FO}^{2}$, $\Sigma_{2}, \Pi_{2}$, and $\Delta_{2}=\Sigma_{2} \cap \Pi_{2}$ :

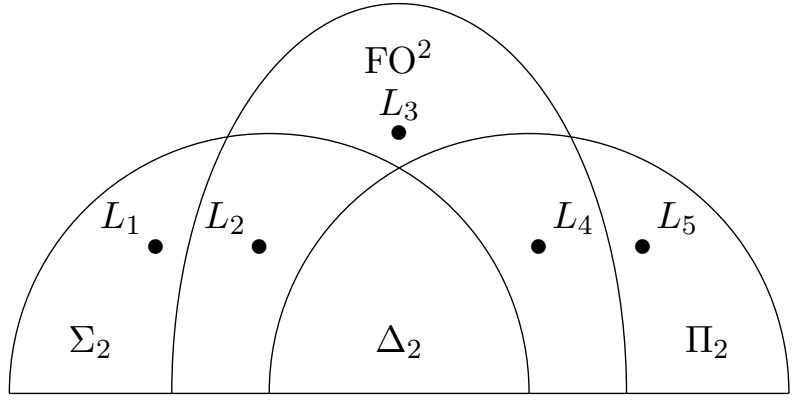

$$
\begin{aligned}
& \text { Here } \Gamma=\{a, b, c\} \text { and } \\
& \begin{aligned}
L_{1} & =\text { "there exists a factor } a b " \\
& =\Gamma^{*} a b \Gamma^{\infty} \\
L_{2} & =\text { "finitely many } a \text { 's" } \\
L_{3}= & \text { "finitely many a's and infinitely } \\
& \text { many b's" }=L_{2} \cap L_{4} \\
L_{4}= & \text { "infinitely many } b \text { 's" } \\
L_{5}= & \text { "there is no factor } a b "=\Gamma^{\infty} \backslash L_{1}
\end{aligned}
\end{aligned}
$$

It will turn out that $L_{4}$ is the closure of $L_{3}$ within some alphabetic topology, whereas $L_{2}$ is not the interior of $L_{3}$ since $L_{3} \subsetneq L_{2}$. In fact, the interior of $L_{3}$ with respect to our topology is empty.

For basic notions on languages of infinite words we refer to standard references such as $[8,16]$.

\section{Preliminaries}

Words. Throughout, $\Gamma$ is a finite alphabet, $A \subseteq \Gamma$ is a subset of the alphabet, $u, v, w$ are finite words, and $\alpha, \beta, \gamma$ are finite or infinite words. If not specified otherwise, then in all examples we assume that $\Gamma$ has three different letters $a, b, c$. By $u \leq \alpha$ we mean that $u$ is a (finite) prefix of $\alpha$. By $\operatorname{alph}(\alpha)$ we denote the alphabet of $\alpha$, i.e., the letters occurring in the sequence $\alpha$. As usual, $\Gamma^{*}$ is the free monoid of finite words over $\Gamma$. The neutral element is the empty word 1. If $L$ is a subset of a monoid, then $L^{*}$ is the submonoid generated by $L$. For $L \subseteq \Gamma^{*}$ we let $L^{\omega}=\left\{u_{1} u_{2} \cdots \mid u_{i} \in L\right.$ for all $\left.i \geq 1\right\}$ be the set of infinite products. We also let $L^{\infty}=L^{*} \cup L^{\omega}$. A natural convention is $1^{\omega}=1$. Thus, $L^{\infty}=L^{\omega}$ if and only if $1 \in L$. 
We write $\operatorname{im}(\alpha)$ for those letters in $\operatorname{alph}(\alpha)$ which have infinitely many different occurrences in $\alpha$. The notation has been introduced in the framework of so called complex traces, see e.g. [6] for a detailed discussion of this concept. The notation $\operatorname{im}(\alpha)$ refers to the imaginary part and we adopt it here. A crucial role for us are sets of the form $A^{\mathrm{im}}$, where, by definition, $A^{\mathrm{im}}$ is the set of words $\alpha$ such that $\operatorname{im}(\alpha)=A$. Note that $\Gamma^{*}=\emptyset^{\mathrm{im}}$. The set $\Gamma^{\infty}$ is the disjoint union over all $A^{\mathrm{im}}$.

Logic and regular sets. We assume that the reader is familiar with basic concepts in formal language theory. All languages $L$ here can be assumed to be regular. The finite part $L \cap \Gamma^{*}$ can be assumed to be specified by some NFA and infinite part $L \cap \Gamma^{\omega}$ can be assumed to be specified by some Bchi automaton. We focus on regular languages which are given by first-order sentences in $\mathrm{FO}[<]$. Thus, atomic predicates are $\lambda(x)=a$ and $x<y$ saying that position $x$ in a word $\alpha$ is labeled with $a \in \Gamma$ and position $x$ is less than $y$, respectively. By $\mathrm{FO}^{2}$ we mean $\mathrm{FO}[<]$-sentences which use at most two names $x$ and $y$ as variables or the class of languages specified by such formulas. Similarly, $\Sigma_{2}$ means $\mathrm{FO}[<]$-sentences which are in prenex normal form and which start with a block of existential quantifiers, followed by a block of universal quantifiers and a Boolean combination of atomic formulas. A $\Pi_{2}$-formula means a negation of a $\Sigma_{2}$-formula. The notations $\Sigma_{2}$ and $\Pi_{2}$ refer also to the corresponding language classes. The class $\Delta_{2}$ means the class of $\Sigma_{2}$-formulas which have an equivalent $\Pi_{2^{-}}$ formula. But the notion of equivalence depends on the set of models we use. If the models are finite words, then a result of Thérien and Wilke [14] states $\mathrm{FO}^{2}=\Delta_{2}$. Moreover, $\mathrm{FO}^{2}$ is the class of regular languages in $\Gamma^{*}$ which are recognized by some finite monoid in the variety DA and a classical result of Schützenberger shows that DA also coincides with unambiguous polynomials [10]. We refer to $[12,4]$ for more background on the class DA. It is a class of finite monoids defined e.g. by equations of type $(x y)^{\omega}=(x y)^{\omega} y(x y)^{\omega}$. We recall that the class DA can also be defined by equations of the form $e=e s e$ for all idempotents $e$ (i.e., $e^{2}=e$ ) and for all $s$ generated by factors of $e$, see e.g. [17].

Saying that formulas are equivalent if they agree on all finite and infinite words changes the picture. This is actually the starting point of this work. So, in this paper models are finite and infinite words. We are mainly interested in infinite words, but it does no harm to include finite words, and this makes the situation more uniform and the results on finite words reappear as special cases. See e.g. Theorem 8.1 which means $\mathrm{FO}^{2}=\Delta_{2}$ for finite words by choosing $A=\emptyset$. An important concept in this paper is topology.

\section{The alphabetic topology and polynomials}

We equip $\Gamma^{\infty}$ with a refinement of the usual Cantor topology. The languages $u \Gamma^{\infty}$ form a basis of the Cantor topology. As we will see, topological information is crucial in our characterization results. We define the alphabetic topology by its basis, which is given by all sets of the form $u A^{\infty}$. Thus, a set $L$ is open if and only if for each $A \subseteq \Gamma$ there is a set of finite words $W_{A} \subseteq \Gamma^{*}$ such that $L=\bigcup W_{A} A^{\infty}$. By definition, a set is closed, if its complement is open; and it is clopen, if it is both open and closed. All sets $A^{\infty}$ are clopen. A set $A^{\mathrm{im}}$ is not open unless $A=\emptyset$, it is not closed unless $A=\Gamma$.

Remark 3.1. The space $\Gamma^{\infty}$ with the alphabetic topology is Hausdorff, but not compact, in general (in contrast to the Cantor topology). To see that it is not compact for $\Gamma=\{a, b\}$ note that $\Gamma^{\infty}=a^{\omega} \cup \Gamma^{*} b \Gamma^{\infty}$. The singleton set $a^{\omega}$ is clopen, but for no finite subset $F \subseteq \Gamma^{*}$ we have $\Gamma^{\infty}=a^{\omega} \cup F b \Gamma^{\infty}$. 
For a language $L$, its closure $\bar{L}$ is the intersection of all closed sets containing $L$. A word $\alpha \in \Gamma^{\infty}$ belongs to $\bar{L}$ if for all open subsets $U \subseteq \Gamma^{\infty}$ with $\alpha \in U$ we have $U \cap L \neq \emptyset$. The interior of $L$ is the complement of the closure of its complement. For languages $L$ and $K$ we define the right quotient as a language of finite words by $L / K=\left\{u \in \Gamma^{*} \mid u \alpha \in L\right.$ for some $\left.\alpha \in K\right\}$. For $L \subseteq \Gamma^{*}$ we define

$$
\vec{L}=\left\{\alpha \in \Gamma^{\infty} \mid \text { for every prefix } u \leq \alpha \text { there exists } u v \leq \alpha \text { with } u v \in L\right\} \text {. }
$$

Proposition 3.2. In the alphabetic topology we have $\overline{A^{\mathrm{im}}}=\bigcup_{A \subseteq B} B^{\mathrm{im}}$ and

$$
\bar{L}=\bigcup_{A \subseteq \Gamma}\left(\overline{L / A^{\infty}} \cap A^{\mathrm{im}}\right)=\bigcup_{A \subseteq \Gamma}\left(\overline{L / A^{\infty}} \cap \overline{A^{\mathrm{im}}}\right) .
$$

Corollary 3.3. Given a regular language $L \subseteq \Gamma^{\infty}$, we can decide whether $L$ is closed (open resp., clopen resp.).

Actually, we have a more precise statement than pure decidability.

Theorem 3.4. The following problem is PSPACE-complete:

Input: $A$ Bchi automaton $\mathcal{A}$ with $L(\mathcal{A}) \subseteq \Gamma^{\omega}$.

Question: Is the regular language $L(\mathcal{A})$ closed?

Remark 3.5. Neither languages of the form $\overrightarrow{L / A^{\infty}}$ nor $\overrightarrow{L / A^{\infty}} \cap \overline{A^{\text {im }}}$ as in Proposition 3.2 need to be closed. Indeed, let $A=\{a\}, B=\{a, b\}$, and $L=a^{*}(a b)^{*} b a^{\omega}$. Then $L / A^{\infty}=$ $a^{*}(a b)^{*} b a^{*}$ and $L / B^{\infty}$ is the set of all finite prefixes of words in $L$. We have $\overrightarrow{L / A^{\infty}}=$ $a^{*}(a b)^{*} b a^{\infty}$ and $\overrightarrow{L / A^{\infty}} \cap \overline{A^{\mathrm{im}}}=a^{*}(a b)^{*} b a^{\omega}=L$. The language $\overrightarrow{L / A^{\infty}}$ is open but neither $\overrightarrow{L / A^{\infty}}$ nor $\overrightarrow{L / A^{\infty}} \cap \overline{A^{\mathrm{im}}}$ is closed in the alphabetic topology, because $(a b)^{\omega}$ belongs to both closures. We have $\overrightarrow{L / B^{\infty}}=a^{*}(a b)^{*} b a^{\infty} \cup a^{*}(a b)^{\omega}$ and $\overrightarrow{L / B^{\infty}} \cap B^{\mathrm{im}}=a^{*}(a b)^{\omega}$. Both sets are closed. Actually, $\bar{L}=L \cup a^{*}(a b)^{\omega}$ in the alphabetic topology. Finally note that $\bar{L}$ is not closed in the Cantor topology since $a^{\omega} \notin \bar{L}$. Remember that a basis of the Cantor topology are the sets of the form $u \Gamma^{\infty}$.

Frequently we apply the closure operator to polynomials. A polynomial is a finite union of monomials. A monomial (of degree $k$ ) is a language of the form $A_{1}^{*} a_{1} \cdots A_{k}^{*} a_{k} A_{k+1}^{\infty}$ with $a_{i} \in \Gamma$ and $A_{i} \subseteq \Gamma$. In particular, $A_{1}^{*} a_{1} \cdots A_{k}^{*} a_{k}$ is a monomial with $A_{k+1}=\emptyset$. The set $A^{*}$ is a polynomial since $A^{*}=\emptyset^{\infty} \cup \bigcup_{a \in A} A^{*} a$. It is not hard to see that polynomials are closed under intersection. Thus, $A_{1}^{*} a_{1} \cdots A_{k}^{*} a_{k} A_{k+1}^{*}$ is in our language a polynomial, but not a monomial unless $A_{k+1}=\emptyset$. A monomial $P=A_{1}^{*} a_{1} \cdots A_{k}^{*} a_{k} A_{k+1}^{\infty}$ is unambiguous if for every $\alpha \in P$ there exists a unique factorization $\alpha=u_{1} a_{1} \cdots u_{k} a_{k} \beta$ such that $u_{i} \in A_{i}^{*}$ and $\beta \in A_{k+1}^{\infty}$. A polynomial is unambiguous if it is a finite union of unambiguous monomials.

It follows from the definition of the alphabetic topology that polynomials are open. Actually, it is the coarsest topology with this property. The crucial observation is that we have a syntactic description of the closure of a polynomial as a finite union of other polynomials. For later use we make a more precise statement.

Lemma 3.6. Let $P=A_{1}^{*} a_{1} \cdots A_{k}^{*} a_{k} A_{k+1}^{\infty}$ be a monomial and $L=P \cap B^{\text {im }}$ for some $B \subseteq A_{k+1}$. Then the closure of $L$ is given by

$$
\bigcup_{\left\{a_{i}, \ldots, a_{k}\right\} \cup B \subseteq A \subseteq A_{i}} A_{1}^{*} a_{1} \cdots A_{i-1}^{*} a_{i-1} A_{i}^{\infty} \cap A^{\mathrm{im}} .
$$


Proof. First consider an index $i$ with $1 \leq i \leq k+1$ such that $\left\{a_{i}, \ldots, a_{k}\right\} \cup B \subseteq A \subseteq A_{i}$. Let $\alpha \in A_{1}^{*} a_{1} \cdots A_{i-1}^{*} a_{i-1} A_{i}^{\infty} \cap A^{\mathrm{im}}$. We have to show that $\alpha$ is in the closure of $L$. Let $\alpha=u \beta$ with $u \in A_{1}^{*} a_{1} \cdots A_{i-1}^{*} a_{i-1} A_{i}^{*}$ and $\beta \in A^{\infty} \cap A^{\mathrm{im}}$. We show that $u A^{\infty} \cap L \neq \emptyset$. Choose some $\gamma \in B^{\infty} \cap B^{\mathrm{im}}$. As $B \subseteq A_{k+1}$ holds by hypothesis, we see that $u a_{i} \cdots a_{k} \gamma \in P$, and hence $u a_{i} \cdots a_{k} \gamma \in u A^{\infty} \cap L$.

Let now $\alpha \in \bar{L}$ and write $\alpha \in u v_{1} \cdots v_{k+1} A^{\infty} \cap A^{\text {im }}$ with $\operatorname{alph}\left(v_{j}\right)=A$. There exists $\gamma \in A^{\infty}$ such that $u v_{1} \cdots v_{k+1} \gamma \in P \cap B^{\mathrm{im}}$. This implies $B \subseteq A$. Since $u v_{1} \cdots v_{k+1} \gamma \in$ $A_{1}^{*} a_{1} \cdots A_{k}^{*} a_{k} A_{k+1}^{\infty}$ there are some $1 \leq i, j \leq k+1$ such that $u v_{1} \cdots v_{j-1}$ belongs to $A_{1}^{*} a_{1} \cdots A_{i-1}^{*} a_{i-1} A_{i}^{*} \cap A^{\mathrm{im}}, v_{j} \in A_{i}^{*}$, and $v_{j+1} \cdots v_{k+1} \gamma \in A_{i}^{*} a_{i} \cdots A_{k}^{*} a_{k} A_{k+1}^{\infty} \cap A^{\infty}$. Therefore $\left\{a_{i}, \ldots, a_{k}\right\} \subseteq A \subseteq A_{i}$, too. It follows that $\alpha \in A_{1}^{*} a_{1} \cdots A_{i-1}^{*} a_{i-1} A_{i}^{\infty} \cap A^{\mathrm{im}}$.

\section{Recognizability by finite monoids}

By $M$ we denote a finite monoid. We always assume that $M$ is equipped with a partial order $\leq$ being compatible with the multiplication, i.e., $u \leq v$ implies sut $\leq$ svt for all $s, t, u, v \in M$. If not specified otherwise, we may choose $\leq$ to be the identity relation.

For an idempotent element $e \in M$ we define $M_{e}=\{s \in M \mid e \in M s M\}^{*}$. By definition, $M_{e}$ is a submonoid of $M$. If $M$ is generated by $\Gamma$, then $M_{e}$ is generated by $\{a \in \Gamma \mid e \in M a M\}$. We can think of this set as the maximal alphabet of the idempotent $e$. We say that an idempotent $e$ is locally top (locally bottom, resp.) if ese $\leq e$ (ese $\geq e$, resp.) for all $s \in M_{e}$. By DA we denote the class of finite monoids such that $e s e=e$ for all idempotents $e \in M$ and all $s \in M_{e}$. More information about this variant to define DA can be found in [17].

Let $L \subseteq \Gamma^{\infty}$ be a language. The syntactic preorder $\leq_{L}$ over $\Gamma^{*}$ is defined as follows. We let $u \leq_{L} v$ if for all $x, y, z \in \Gamma^{*}$ we have both implications:

$$
x v y z^{\omega} \in L \Rightarrow x u y z^{\omega} \in L \quad \text { and } \quad x(v y)^{\omega} \in L \Rightarrow x(u y)^{\omega} \in L .
$$

Let us recall that $1^{\omega}=1$. Two words $u, v \in \Gamma^{*}$ are syntactically equivalent, written as $u \equiv_{L} v$, if both $u \leq_{L} v$ and $v \leq_{L} u$. This is a congruence and the congruence classes $[u]_{L}=\left\{v \in \Gamma^{*} \mid u \equiv_{L} v\right\}$ form the syntactic monoid $\operatorname{Synt}(L)$ of $L$. The preorder $\leq_{L}$ on words induces a partial order $\leq_{L}$ on congruence classes, and $\left(\operatorname{Synt}(L), \leq_{L}\right)$ becomes an ordered monoid. It is a well-known classical result that the syntactic monoid of a regular language $L \subseteq \Gamma^{\infty}$ is finite, see e.g. $[8,16]$. Moreover, in this case $L$ can be written as a finite union of languages of type $[u]_{L}[v]_{L}^{\omega}$ where $u, v \in \Gamma^{*}$ with $u v \equiv_{L} u$ and $v^{2} \equiv_{L} v$.

Now, let $h: \Gamma^{*} \rightarrow M$ be any surjective homomorphism onto a finite ordered monoid $M$ and let $L \subseteq \Gamma^{\infty}$. If the reference to $h$ is clear, then we denote by $[s]$ the set of finite words $h^{-1}(s)$ for $s \in M$. The following notations are used:

- $(s, e) \in M \times M$ is a linked pair, if $s e=s$ and $e^{2}=e$.

- $h$ weakly recognizes $L$, if

$$
L=\bigcup\left\{[s][e]^{\omega} \mid(s, e) \text { is a linked pair and }[s][e]^{\omega} \subseteq L\right\}
$$

- $h$ strongly recognizes $L$ (or simply recognizes $L$ ), if

$$
L=\bigcup\left\{[s][e]^{\omega} \mid(s, e) \text { is a linked pair and }[s][e]^{\omega} \cap L \neq \emptyset\right\}
$$

- $L$ is downward closed (on finite prefixes) for $h$, if $[s][e]^{\omega} \subseteq L$ implies $[t][e]^{\omega} \subseteq L$ for all $s, t, e \in M$ where $t \leq s$. 
Lemma 4.1. Let $L \subseteq \Gamma^{\infty}$ be a regular language and let $h_{L}: \Gamma^{*} \rightarrow \operatorname{Synt}(L)$ be its syntactic homomorphism. Then for all $s, t, e, f \in M$ such that $t \leq s, f \leq e$, and $[s][e]^{\omega} \subseteq L$ we have $[t][f]^{\omega} \subseteq L$. In particular, $L$ is downward closed (on finite prefixes) for $h_{L}$.

Proof. Let $u \in[s], x \in[e]$ and let $v \in[t], y \in[f]$. Now, $u x^{\omega} \in L$ implies $v x^{\omega} \in L$, which in turn implies $v y^{\omega} \in L$. Since $L$ is regular, $h_{L}$ strongly recognizes $L$, and we obtain $[t][f]^{\omega} \subseteq L$ because $v y^{\omega} \in[t][f]^{\omega} \cap L$.

For lack of space and in order to avoid too much machinery we do not treat $\omega$-semigroups $[9,19]$ in this extended abstract. However, let us define $t f^{\omega} \leq_{L} s e^{\omega}$ for linked pairs by the implication:

$$
[s][e]^{\omega} \subseteq L \Rightarrow[t][f]^{\omega} \subseteq L
$$

With this notation we can give an algebraic characterization of being open.

Lemma 4.2. A regular language $L \subseteq \Gamma^{\infty}$ is open in the alphabetic topology if and only if for all linked pairs $(s, e),(t, f)$ of $M=\operatorname{Synt}(L)$ with $t, f \in M_{e}$ we have st $f^{\omega} \leq_{L} s^{\omega}$.

\section{The fragment $\Sigma_{2}$}

By a (slight extension of a) result of Thomas [15] on $\omega$-languages we know that a language $L \subseteq \Gamma^{\infty}$ is definable in $\Sigma_{2}$ if and only if $L$ is a polynomial. However, this statement alone does not yield decidability. It turns out that we obtain decidability by a combination of an algebraic and a topological criterion. This decidability result has also been shown by Bojańczyk [2] using different techniques. We know that polynomials are open. Therefore, we concentrate on algebra.

Lemma 5.1. If $L \subseteq \Gamma^{\infty}$ is a polynomial, then all idempotents of $\operatorname{Synt}(L)$ are locally top.

Theorem 5.2. Let $L \subseteq \Gamma^{\infty}$ be a regular language. The following assertions are equivalent:

(1) $L$ is $\Sigma_{2}$-definable.

(2) $L$ is a polynomial.

(3) $L$ is open in the alphabetic topology and all idempotents of $\operatorname{Synt}(L)$ are locally top.

(4) The syntactic monoid $M=\operatorname{Synt}(L)$ and the syntactic order $\leq_{L}$ satisfy:

(a) For all linked pairs $(s, e),(t, f)$ with $t, f \in M_{e}$ we have stf ${ }^{\omega} \leq_{L} s e^{\omega}$.

(b) $e=e^{2}$ and $s \in M_{e}$ implies ese $\leq_{L} e$.

(5) The following three conditions hold for some homomorphism $h: \Gamma^{*} \rightarrow M$ which weakly recognizes $L$ :

(a) $L$ is open in the alphabetic topology.

(b) All idempotents of $M$ are locally top.

(c) $L$ is downward closed (on finite prefixes) for $h$.

Proof. " $1 \Leftrightarrow 2$ ": This is a slight modification of a result by Thomas [15].

"2 $\Rightarrow 3$ ": By definition, polynomials are open in the alphabetic topology. In Lemma 5.1 it has been shown that all idempotent elements are locally top.

" $3 \Leftrightarrow 4$ ": The equivalence of $L$ being open and " $4 \mathrm{a}$ " is Lemma 4.2. Property " $4 \mathrm{~b}$ " is the definition of all elements being locally top.

"4 $\Rightarrow$ ": Let $h=h_{L}$ be the syntactic homomorphism onto the syntactic monoid $M=$ Synt $(L)$. Applying Lemma 4.2, property "5a" follows from "4a" and " $5 \mathrm{~b}$ " trivially follows from " $4 \mathrm{~b}$ ". The condition "5c" holds for $\operatorname{Synt}(L)$ by Lemma 4.1. 
"5 $\Rightarrow 2$ ": Consider $\alpha \in L$ with $\operatorname{im}(\alpha)=A$. By " $5 \mathrm{a}$ " the language $L$ is open. Hence, there exists a prefix $u$ of $\alpha$ such that $\alpha \in u A^{\infty} \subseteq L$. From the case of finite words and the hypothesis " $5 \mathrm{~b}$ " on $M$, we know that $P=\left\{v \in \Gamma^{*} \mid h(v) \leq h(u)\right\}$ is a polynomial. We can assume that all monomials in $P$ end with a letter. We define the polynomial $P_{\alpha}=P A^{\infty}$. Clearly, $L \subseteq \bigcup\left\{P_{\alpha} \mid \alpha \in L\right\}$ and this union is finite since $M$ is finite. It remains to show that $P_{\alpha} \subseteq L$ for $\alpha \in L$. Let $v \in P$ and $\beta \in A^{\infty}$. We know $u \beta \in L$ and there exists a linked pair $(s, e)$ such that $u \beta \in[s][e]^{\omega} \subseteq L$. Now, there exists $w \gamma=\beta$ such that $u w \in[s]$ and $\gamma \in[e]^{\omega}$. By definition of $P$, we have $h(v) \leq h(u)$ and therefore $t=h(v w) \leq h(u w)=s$. It follows $v \beta=v w \gamma \in[t][e]^{\omega} \subseteq L$ by " 5 c". This shows $P_{\alpha} \subseteq L$ and thus $L=\bigcup\left\{P_{\alpha} \mid \alpha \in L\right\}$.

Corollary 5.3. It is decidable whether a regular language is $\Sigma_{2}$-definable.

Remark 5.4. An $\omega$-language $L \subseteq \Gamma^{\omega}$ is $\Sigma_{2}$-definable, if $L=\left\{\alpha \in \Gamma^{\omega} \mid \alpha \models \varphi\right\}$ for some $\varphi \in \Sigma_{2}$. This is equivalent with $L \cup \Gamma^{*}$ being $\Sigma_{2}$-definable as a subset of $\Gamma^{\infty}$. Thus, the decidability of Corollary 5.3 transfers to $\omega$-regular languages.

\section{Two variable first-order logic}

Etessami, Vardi, and Wilke have given a characterization of $\mathrm{FO}^{2}$ in terms of unary temporal logic [5]. In the same paper, they considered the satisfiability problem for $\mathrm{FO}^{2}$. In this section, we continue the study of $\mathrm{FO}^{2}$ over infinite words.

The following lemma can be proved essentially in the same way as for finite words. The result is also (implicitly) stated in the habilitation thesis of Wilke [20].

Lemma 6.1. Let $L \subseteq \Gamma^{\infty}$ be $\mathrm{FO}^{2}$-definable. Then the syntactic monoid $\operatorname{Synt}(L)$ is in $\mathbf{D A}$.

A set like $A^{\mathrm{im}}$ is $\mathrm{FO}^{2}$-definable, but it is neither open nor closed in the alphabetic topology, in general. Therefore, we need a refinement of the alphabetic topology. As a basis for the strict alphabetic topology we take all sets of the form $u A^{\infty} \cap A^{\mathrm{im}}$. Thus, more sets are open (and closed) than in the alphabetic topology. Another way to define the strict alphabetic topology is to say that it is the coarsest topology on $\Gamma^{\infty}$ where all sets of the form $A_{1}^{*} a_{1} \cdots A_{k}^{*} a_{k} A_{k+1}^{\infty} \cap B^{\mathrm{im}}$ are open. The strict alphabetic topology is not used outside this section, but it is essential here in order to prove the converse of Lemma 6.1.

Lemma 6.2. If $L \subseteq \Gamma^{\infty}$ is strongly recognized by some homomorphism $h: \Gamma^{*} \rightarrow M \in \mathbf{D A}$, then $L$ is clopen in the strict alphabetic topology.

Proof. Since $h$ also strongly recognizes $\Gamma^{\infty} \backslash L$ as well, it is enough to show that $L$ is open. Let $\alpha \in L$ with $\alpha \in[s][e]^{\omega}$ for some linked pair $(s, e)$ and let $A=\operatorname{im}(\alpha)$. We show that $[s] A^{\infty} \cap A^{\mathrm{im}} \subseteq L$. Indeed, let $\beta \in[s] A^{\infty} \cap A^{\mathrm{im}}$. Then we have $\beta=u v \gamma$ with $h(u)=s$, $h(v)=r, \gamma \in[f]^{\omega}$ where $v \in A^{*}, \operatorname{alph}(\gamma)=\operatorname{im}(\gamma)=A$, and $(r, f)$ is a linked pair. Since $M \in \mathbf{D A}$, we obtain $s=s e=\operatorname{ser} f e=\operatorname{srfe}$ and $e f e=e$ and $f e f=f$. Since $h$ strongly recognizes $L$, we can compute as follows:

$$
\beta \in[s r][f]^{\omega}=[s r][f e f]^{\omega}=[\operatorname{srfe}][e f e]^{\omega}=[s][e]^{\omega} \subseteq L
$$

In particular, $\beta \in L$. 
Lemma 6.3. If $L$ is closed in the strict alphabetic topology and if $L$ is weakly recognized by some homomorphism $h: \Gamma^{*} \rightarrow M \in \mathbf{D A}$, then $L$ is a finite union of languages $A_{1}^{*} a_{1} \cdots A_{k}^{*} a_{k} A_{k+1}^{\infty} \cap A_{k+1}^{\mathrm{im}}$, where each $A_{1}^{*} a_{1} \cdots A_{k}^{*} a_{k} A_{k+1}^{\infty}$ is an unambiguous monomial.

Proof. Let $\alpha \in L$. Write $\alpha=u \beta$ with $\beta \in A^{\infty} \cap A^{\text {im }}$ for some $A \subseteq \Gamma$. There is a linked pair $(s, e)$ with $\alpha \in[s][e]^{\omega} \subseteq L$ and we may assume $h(u)=s$ and $\beta \in[e]^{\omega}$. For $A=\emptyset$ we have $[s] \subseteq L$ and, using our knowledge about the finite case, we may include $[s]$ in our finite union of unambiguous polynomials. Therefore, let $A \neq \emptyset$. We may choose an unambiguous monomial $P=A_{1}^{*} a_{1} \cdots A_{k}^{*} a_{k} \subseteq[s]$ such that $u \in P$ and each last position of every letter $a \in\left\{a_{1}, \ldots, a_{k}\right\} \cup A_{1} \cup \cdots \cup A_{k}$ occurs explicitly as some $a_{j}$ in the expression $P$. Note that $[s]$ is a finite union of such monomials. Moreover, we may assume that $u v \in P$ for infinitely many prefixes $v \leq \beta$. Each such $u v$ can uniquely be written as $u v=v_{1} a_{1} \cdots v_{k} a_{k}$ with $v_{i} \in A_{i}^{*}$. This yields a vector in $\mathbb{N}^{k}$ by $\left(\left|v_{1} a_{1}\right|,\left|v_{1} a_{1} v_{2} a_{2}\right|, \ldots,\left|v_{1} a_{1} \cdots v_{k} a_{k}\right|\right)$ for every $u v \in P$. By Dickson's Lemma [3], we may assume that this vector is in no component decreasing when $v$ gets longer. Hence (after removing finitely many $v$ 's) we may assume there is some $i$ such that $\left|v_{1} a_{1} \cdots v_{i} a_{i}\right|$ is constant and $\left|v_{1} a_{1} \cdots v_{i} a_{i} v_{i+1} a_{i+1}\right|$ is strictly increasing. It follows that we may assume $\left\{a_{i+1}, \ldots, a_{k}\right\} \subseteq \operatorname{alph}\left(v_{i+1}\right)=A \subseteq A_{i+1}$. In particular, $\alpha \in A_{1}^{*} a_{1} \cdots A_{i}^{*} a_{i} A^{\infty} \cap A^{\mathrm{im}}$. It is clear that this expression is unambiguous.

It remains to show $A_{1}^{*} a_{1} \cdots A_{i}^{*} a_{i} A^{\infty} \cap A^{\text {im }} \subseteq L$. Consider $u^{\prime} \gamma$ with $u^{\prime} \in A_{1}^{*} a_{1} \cdots A_{i}^{*} a_{i}$ and $\gamma \in A^{\infty} \cap A^{\mathrm{im}}$. Since $L$ is closed, it is enough to show that $u^{\prime} \gamma$ belongs to the closure of $L$ in the strict alphabetic topology. Choose any prefix $w \leq \gamma$. It is enough to show that $u^{\prime} w A^{\infty} \cap A^{\mathrm{im}} \cap L \neq \emptyset$. Let $z \in \Gamma^{*}$ with $\operatorname{alph}(z)=A$ and $h(z)=e$. Since $w \in A^{*} \subseteq A_{i+1}^{*}$, we have $u^{\prime} w a_{i+1} \cdots a_{k} \in P \subseteq[s]$. Hence $u^{\prime} w a_{i+1} \cdots a_{k} z^{\omega} \in[s][e]^{\omega} \subseteq L$.

Lemma 6.4. Every language $A^{\mathrm{im}}$ and every unambiguous monomial $A_{1}^{*} a_{1} \cdots A_{k}^{*} a_{k} A_{k+1}^{\infty}$ is $\mathrm{FO}^{2}$-definable.

Theorem 6.5. Let $L \subseteq \Gamma^{\infty}$. The following assertions are equivalent:

(1) $L$ is $\mathrm{FO}^{2}$-definable.

(2) $L$ is regular and $\operatorname{Synt}(L) \in \mathbf{D A}$.

(3) $L$ is strongly recognized by some homomorphism $h: \Gamma^{*} \rightarrow M \in \mathbf{D A}$.

(4) $L$ is closed in the strict alphabetic topology and $L$ is weakly recognized by some homomorphism $h: \Gamma^{*} \rightarrow M \in \mathbf{D A}$.

(5) $L$ is a finite union of sets of the form $A_{1}^{*} a_{1} \cdots A_{k}^{*} a_{k} A_{k+1}^{\infty} \cap A_{k+1}^{\mathrm{im}}$, where each language $A_{1}^{*} a_{1} \cdots A_{k}^{*} a_{k} A_{k+1}^{\infty}$ is an unambiguous monomial.

Proof. " $1 \Rightarrow 2$ ": First-order definable languages are regular; $\operatorname{Synt}(L) \in \mathbf{D A}$ by Lemma 6.1. "2 $\Rightarrow 3$ ": Trivial, since $\operatorname{Synt}(L)$ strongly recognizes $L$. "3 $\Rightarrow 4$ ": Strong recognition implies weak recognition; closure in the strict alphabetic topology follows by Lemma 6.2. " $4 \Rightarrow 5$ ": Lemma 6.3. "5 $\Rightarrow 1$ ": Lemma 6.4.

Recall that if a language $L \subseteq \Gamma^{\infty}$ is weakly recognizable by a finite monoid, then it is also strongly recognizable by a finite monoid. The same holds for aperiodic monoids, but Theorem 6.5 suggests that this fails for DA. Indeed, we have the following example.

Example 6.6. Let $\Gamma=\{a, b, c\}$. Consider the congruence of finite index such that each class $[u]$ is defined by the set of words $v$ where $u$ and $v$ agree on all suffixes of length at most 2. The quotient monoid of $\Gamma^{*}$ by this congruence is in DA. Let $L=[a b]^{\omega}=\left(\Gamma^{*} a b\right)^{\omega}$. Then, by definition, $L$ is weakly recognizable in DA. But $L$ is the language of all $\alpha$ which contain infinitely many factors of the form $a b$. This is however not closed for the strict alphabetic 
topology since $(a c b)^{\omega} \notin L$, but $(a c b)^{\omega}$ belongs to the strict alphabetic closure of $L$ since every open set $U$ with $(a c b)^{\omega} \in U$ contains some $(a c b)^{m}(c a b)^{\omega}$ and $\left[(a c b)^{m}(c a b)\right]=[a b]$ for all $m \geq 0$.

\section{Unambiguous polynomials and the fragments $\mathrm{FO}^{2} \cap \Sigma_{2}$ and $\mathrm{FO}^{2} \cap \Pi_{2}$}

Theorem 7.1. Let $L \subseteq \Gamma^{\infty}$. The following assertions are equivalent:

(1) $L$ is both $\mathrm{FO}^{2}$-definable and $\Sigma_{2}$-definable.

(2) $L$ is $\mathrm{FO}^{2}$-definable and open in the alphabetic topology.

(3) $L$ is a finite union of unambiguous monomials of the form $A_{1}^{*} a_{1} \cdots A_{k}^{*} a_{k} A_{k+1}^{\infty}$.

(4) $L$ is the interior of some $\mathrm{FO}^{2}$-definable language.

Theorem 7.2. Let $L \subseteq \Gamma^{\infty}$ be a regular language. The following assertions are equivalent:

(1) $L$ is both $\mathrm{FO}^{2}$-definable and $\Pi_{2}$-definable.

(2) $L$ is $\mathrm{FO}^{2}$-definable and closed in the alphabetic topology.

(3) $L$ is the closure of some $\mathrm{FO}^{2}$-definable language.

Theorem 7.2 is not fully satisfactory since we do not have any direct characterization in terms of polynomials. We might wish that if $L$ is closed (and $L \in \Pi_{2} \cap \mathrm{FO}^{2}$ ), then it is a finite union of languages $K \cap B^{\mathrm{im}}$ where each $K \cap B^{\mathrm{im}}$ is closed. But this is not true: Let $L=\Gamma^{*} a \cup \Gamma^{\omega}$, then $L$ is closed and in $\Pi_{2} \cap \mathrm{FO}^{2}$, but cannot be written in this form because $L=\Gamma^{*} a$ is not closed. We also note that the closure of a language $L$ in $\mathrm{FO}^{2} \cap \Sigma_{2}$ needs not to be in $\Delta_{2}$. A counter-example is the language $L=\Gamma^{*} a b c$. By Lemma 3.6, the closure of $L$ is $\bar{L}=L \cup \Gamma^{\mathrm{im}}$ which is not $\Sigma_{2}$-definable.

\section{The fragment $\Delta_{2}=\Sigma_{2} \cap \Pi_{2}$}

For finite words we have the well-known theorem that $\mathrm{FO}^{2}$-definability is equivalent to $\Delta_{2}$-definability. However, this does not transfer to $\omega$-words where $\Delta_{2}$ forms a proper subclass of $\mathrm{FO}^{2}$. Consider $L=\{a, b\}^{\mathrm{im}}$, then $L$ is neither open nor closed, in general. Hence $L \in \mathrm{FO}^{2} \backslash\left(\Sigma_{2} \cup \Pi_{2}\right)$. The result for finite words is therefore somewhat misleading. The correct translation for the general case is:

Theorem 8.1. For all $A \subseteq \Gamma$ the following assertions are equivalent:

(1) $L \cap A^{\mathrm{im}}$ is $\mathrm{FO}^{2}$-definable.

(2) There are languages $L_{\sigma} \in \mathrm{FO}^{2} \cap \Sigma_{2}$ and $L_{\pi} \in \mathrm{FO}^{2} \cap \Pi_{2}$ such that

$$
L \cap A^{\mathrm{im}}=L_{\sigma} \cap A^{\mathrm{im}}=L_{\pi} \cap A^{\mathrm{im}} .
$$

(3) There are languages $L_{\sigma} \in \Sigma_{2}$ and $L_{\pi} \in \Pi_{2}$ such that

$$
L \cap A^{\mathrm{im}}=L_{\sigma} \cap A^{\mathrm{im}}=L_{\pi} \cap A^{\mathrm{im}} .
$$

Note that we cannot expect that $L_{\sigma}=L_{\pi}$ in the statement above, because $L_{\sigma}$ is open and $L_{\pi}$ is closed. Hence, a language in $\Delta_{2}$ must be clopen. The first step for a convenient characterization on $\Delta_{2}$ is therefore a description of clopen unambiguous monomials. 
Lemma 8.2. Let $P=A_{1}^{*} a_{1} \cdots A_{k}^{*} a_{k} A^{\infty}$ be an unambiguous monomial. The following assertions are equivalent:

(1) There is no $1 \leq i \leq k$ such that $\left\{a_{i}, \ldots, a_{k}\right\} \subseteq A_{i}$.

(2) $P$ is closed in the alphabetic topology.

(3) $P$ is clopen in the alphabetic topology.

Proof. " $1 \Rightarrow 2$ ": For a moment let $A_{k+1}=A$. By Lemma 3.6 we know that the closure of $P$ is:

$$
\bigcup_{\left\{a_{i}, \ldots, a_{k}\right\} \subseteq B \subseteq A_{i}} A_{1}^{*} a_{1} \cdots A_{i-1}^{*} a_{i-1}\left(A_{i}^{\infty} \cap B^{\mathrm{im}}\right) .
$$

Since there is no $\left\{a_{i}, \ldots, a_{k}\right\} \subseteq A_{i}$ for $1 \leq i \leq k$, we see that this union is just $P$ itself. Therefore, $P$ is closed. " $2 \Rightarrow 3$ ": is clear, because $P$ is open. " $3 \Rightarrow 1$ ": Assume by contradiction that $\left\{a_{i}, \ldots, a_{k}\right\} \subseteq A_{i}$ for some $1 \leq i \leq k$. We have $a_{1} \cdots a_{i-1}\left(a_{i} \cdots a_{k}\right)^{m} \in P$ for all $m \geq 1$. As $P$ is closed we see $a_{1} \cdots a_{i-1}\left(a_{i} \cdots a_{k}\right)^{\omega} \in P$ and hence $\left\{a_{i}, \ldots, a_{k}\right\} \subseteq A$. But this is a contradiction to the fact that $P$ is unambiguous since $\left\{a_{i}, \ldots, a_{k}\right\} \subseteq A_{i} \cap A$ implies that $a_{1} \cdots a_{i-1}\left(a_{i} \cdots a_{k}\right)^{2} \in P$ has two different factorizations.

Lemma 8.3. Let $L \subseteq \Gamma^{\infty}$ be a closed polynomial. For every unambiguous monomial $P=A_{1}^{*} a_{1} \cdots A_{k}^{*} a_{k} A^{\infty} \subseteq L$ there exist closed unambiguous monomials $Q_{1}, \ldots, Q_{\ell}$ such that $P \subseteq Q_{1} \cup \cdots \cup Q_{\ell} \subseteq L$, i.e., there exists a finite covering of $P$ with closed unambiguous monomials in $L$.

Theorem 8.4. Let $L \subseteq \Gamma^{\infty}$. The following assertions are equivalent.

(1) $L$ is $\Delta_{2}$-definable.

(2) $L$ is $\mathrm{FO}^{2}$-definable and $L$ is clopen in the alphabetic topology.

(3) $L$ is a finite union of unambiguous closed monomials $A_{1}^{*} a_{1} \cdots A_{k}^{*} a_{k} A^{\infty}$, i.e., there is no $1 \leq i \leq k$ such that $\left\{a_{i}, \ldots, a_{k}\right\} \subseteq A_{i}$.

(4) $L$ is regular, $\operatorname{Synt}(L) \in \mathbf{D A}$, and for all linked pairs $(s, e),(t, f)$ with $s \mathcal{R} t$ (i.e., there exist $x, y \in \operatorname{Synt}(L)$ such that $s=t x$ and $t=$ sy) we have

$$
[s][e]^{\omega} \subseteq L \Leftrightarrow[t][f]^{\omega} \subseteq L .
$$

Proof. "1 $\Rightarrow 2$ ": By Theorem 5.2 and its dual version for $\Pi_{2}$, we see that $\operatorname{Synt}(L) \in \mathbf{D A}$ and that $L$ is clopen in the alphabetic topology. From Theorem 6.5 it follows that $L$ is $\mathrm{FO}^{2}$-definable. " $2 \Rightarrow 3$ ": By Theorem $7.1, L$ is a finite union of unambiguous monomials. Property "3" now follows by Lemma 8.3 and Lemma 8.2. "3 $\Rightarrow 1$ ": Theorem 7.1 and Theorem 7.2.

"2 $\Rightarrow 4$ ": By Theorem 6.5, we see that $\operatorname{Synt}(L) \in$ DA. Suppose $[s][e]^{\omega} \subseteq L$ and let $s=t x$ and $t=s y$. Since $L$ is closed we see that $[s][e y f x]^{\omega} \subseteq L$ and by strong recognition we conclude $[t][\text { fxey }]^{\omega} \subseteq L$. Let $A=\bigcup\{\operatorname{alph}(v) \mid v \in[f]\}$. Since $L$ is open and by strong recognition, there exists $r \in \mathbb{N}$ such that $[t][f x e y]^{r} A^{\infty} \subseteq L$. Moreover, $t=t f x e y$ and thus, $[t] A^{\infty} \subseteq L$. In particular, $[t][f]^{\omega} \subseteq L$ because $[f] \subseteq A^{*}$.

" $4 \Rightarrow 2$ ": Definability in $\mathrm{FO}^{2}$ follows by Theorem 6.5. By symmetry, it suffices to show that $L$ is open. Let $\alpha \in[s][e]^{\omega} \subseteq L$ for some linked pair $(s, e)$ and write $\alpha=u \beta$ with $u \in[s]$ and $\beta \in[e]^{\omega} \cap A^{\infty} \cap A^{\mathrm{im}}$ for some $A \subseteq \Gamma$. Let $v \leq \beta$ be a prefix such that $v \in[e]$ and $\operatorname{alph}(v)=\operatorname{alph}(\beta)$. We want to show $u v A^{\infty} \subseteq L$. Consider $u v \gamma \in \Gamma^{\infty}$ where $\gamma \in A^{\infty}$. We have $u v \gamma \in[t][f]^{\omega}$ for some linked pair $(t, f)$. Let $v^{\prime} \leq \gamma$ such that $u v v^{\prime} \in[t]$. Since $\operatorname{Synt}(L) \in \mathbf{D A}$ we have $v v^{\prime} v \in[e]$ and $s=t \cdot h(v)$. Together with $t=s \cdot h\left(v^{\prime}\right)$ it follows $s \mathcal{R} t$ and by " 4 " we obtain $u v \gamma \in[t][f]^{\omega} \subseteq L$. 


\section{Summary}

We gave language-theoretic, algebraic and topological characterizations for several firstorder fragments over infinite words. Since $\mathrm{FO}^{2}$ and $\Delta_{2}$ have the same expressive power only when restricted to some fixed set of letters occurring infinitely often (Thm. 8.1), the picture becomes more complex. By Pol we denote the language class of polynomials, UPol are unambiguous polynomials, and restricted UPol is a proper subclass of UPol. All of the below-mentioned algebraic properties are decidable, since the syntactic monoid of a regular language is effectively computable $[8,16]$. Together with the PSPACE-completeness of the problem whether a language is closed in the alphabetic topology (Thm.3.4), this yields decidability of the membership problem for the respective first-order fragments as a corollary. Decidability was shown before by Wilke [20] for $\mathrm{FO}^{2}$ and by Bojańczyk [2] for $\Sigma_{2}$. Characterizations for the fragment $\Sigma_{1}$ and its Boolean closure over infinite words (using topological notions based on the Cantor topology) are due to Pin [9]; see also [8].

\begin{tabular}{ccrll}
\hline Logic & Languages & Algebra & Topology & \\
\hline$\Sigma_{2}$ & Pol & $e M_{e} e \leq e+\begin{array}{l}\text { open } \\
\text { (alphabetic) }\end{array}$ & Thm. 5.2 \\
\hline $\mathrm{FO}^{2}$ & $\mathrm{UPol}+A^{\text {im }}$ & $\begin{array}{r}\text { strong DA } \\
\text { weak DA }\end{array}$ & $\begin{array}{l}\text { closed } \\
\text { (strict alphabetic) }\end{array}$ & Thm.5 \\
\hline $\mathrm{FO}^{2} \cap \Sigma_{2}$ & UPol & DA $+\begin{array}{l}\text { open } \\
\text { (alphabetic) }\end{array}$ & Thm. 7.1 \\
\hline $\mathrm{FO}^{2} \cap \Pi_{2}$ & & DA $+\begin{array}{l}\text { closed } \\
\text { (alphabetic) }\end{array}$ & Thm. 7.2 \\
\hline$\Delta_{2}$ & restricted UPol & DA $+\begin{array}{l}\text { clopen } \\
\text { (alphabetic) }\end{array}$ & Thm. 8.4 \\
\hline
\end{tabular}

\section{Outlook and open problems}

By definition, $\Sigma_{1}$-definable languages are open in the Cantor topology. We introduced an alphabetic topology such that $\Sigma_{2}$-definable languages are open in this topology. Therefore, an interesting question is whether it is possible to extend this topological approach to higher levels of the first-order alternation hierarchy. To date, even over finite words no decidable characterization of the Boolean closure of $\Sigma_{2}$ is known. In case that a decidable criterion is found, it might lead to a decidable criterion for infinite words simply by adding a condition of the form "clopen in some appropriate topology". Another possible way to generalize our approach might be combinations of algebraic and topological characterizations for fragments with successor predicate suc such as $\mathrm{FO}^{2}\left[<\right.$, suc] or $\Sigma_{2}[<$, suc]. A characterization of those languages which are weakly recognizable by monoids in DA is also open. 


\section{Acknowledgements}

We thank the anonymous referees for many useful suggestions and we thank Luc Dartois from the ENS Cachan for helpful discussions during his internship in Stuttgart and for his firm conviction that $\mathrm{FO}^{2}$ should coincide with DA.

\section{References}

[1] M. Bojańczyk. Two-way unary temporal logic over trees. In LICS'O\%, pages 121-130. IEEE Computer Society, 2007.

[2] M. Bojańczyk. The common fragment of ACTL and LTL. In Foundations of Software Science and Computational Structures, 11th International Conference, FoSSaCS 2008, Proceedings, volume 4962 of Lecture Notes in Computer Science, pages 172-185. Springer-Verlag, 2008.

[3] L. E. Dickson. Finiteness of the odd perfect and primitive abundant numbers with $n$ distinct prime factors. American Journal of Mathematics, 35(4):413-422, 1913.

[4] V. Diekert, P. Gastin, and M. Kufleitner. A survey on small fragments of first-order logic over finite words. International Journal of Foundations of Computer Science, 19(3):513-548, June 2008. Special issue DLT 2007.

[5] K. Etessami, M. Y. Vardi, and Th. Wilke. First-order logic with two variables and unary temporal logic. Information and Computation, 179(2):279-295, 2002.

[6] P. Gastin and A. Petit. Infinite traces. In V. Diekert and G. Rozenberg, editors, The Book of Traces, chapter 11, pages 393-486. World Scientific, Singapore, 1995.

[7] A. R. Meyer and L. J. Stockmeyer. The equivalence problem for regular expressions with squaring requires exponential space. In 13th Annual Symposium on Switching and Automata Theory, pages 125129. IEEE Computer Society, 1972.

[8] D. Perrin and J.-É. Pin. Infinite words, volume 141 of Pure and Applied Mathematics. Elsevier, Amsterdam, 2004.

[9] J.-É. Pin. Positive varieties and infinite words. In C. Lucchesi and A. Moura, editors, Latin'98, volume 1380 of Lecture Notes in Computer Science, pages 76-87. Springer-Verlag, 1998.

[10] M. P. Schützenberger. Sur le produit de concaténation non ambigu. Semigroup Forum, 13:47-75, 1976.

[11] A. P. Sistla, M. Y. Vardi, and P. L. Wolper. The complementation problem for Büchi automata with applications to temporal logic. Theoretical Computer Science, 49(2-3):217-237, 1987.

[12] P. Tesson and D. Thérien. Diamonds are Forever: The Variety DA. In G. M. dos Gomes Moreira da Cunha, P. V. A. da Silva, and J.-É. Pin, editors, Semigroups, Algorithms, Automata and Languages, Coimbra (Portugal) 2001, pages 475-500. World Scientific, 2002.

[13] P. Tesson and D. Thérien. Logic meets algebra: The case of regular languages. Logical Methods in Computer Science, 3(1):1-37, 2007.

[14] D. Thérien and Th. Wilke. Over words, two variables are as powerful as one quantifier alternation. In STOC, pages 234-240, 1998.

[15] W. Thomas. Classifying regular events in symbolic logic. Journal of Computer and System Sciences, 25:360-376, 1982.

[16] W. Thomas. Automata on infinite objects. In J. van Leeuwen, editor, Handbook of Theoretical Computer Science, chapter 4, pages 133-191. Elsevier Science Publishers B. V., 1990.

[17] P. Weil. Some results on the dot-depth hierarchy. Semigroup Forum, 46:352-370, 1993.

[18] P. Weil. Algebraic recognizability of languages. In Mathematical Foundations of Computer Science 2004, volume 3153 of Lecture Notes in Computer Science, pages 149-174. Springer, Berlin, 2004.

[19] Th. Wilke. An Eilenberg theorem for $\infty$-languages. In ICALP'91, volume 510 of Lecture Notes in Computer Science, pages 588-599. Springer-Verlag, 1991.

[20] Th. Wilke. Classifying Discrete Temporal Properties. Habilitationsschrift, Universität Kiel, April 1998. 